\title{
Comments on Acupuncture in Post-Stroke Shoulder Pain Syndrome with Multiple Sclerosis: A Case Study
}

Dear Editor,

I read with interest the case report titled "Acupuncture in Post-Stroke Shoulder Pain Syndrome with Multiple Sclerosis: A Case Study" by Gan and Santorelli [1] and would like to comment regarding the use of acupuncture in central poststroke pain (CPSP).

First, it is an excellent consideration by Gan et al to choose acupuncture as a non-pharmacological modality to treat CPSP. CPSP leads to disabling pain, which can be spontaneous or evoked. It is well-established even in multiple sclerosis without cerebral vascular infarct that the central pain can cause disabling symptoms with a prevalence of approximately $30 \%$ [2]. While the pathophysiology of CPSP remains incompletely understood with central disinhibition and complex involvement of the spinothalamocortical pathway leading to allodynia or dysesthesia, the analgesic role of acupuncture cannot be underestimated [3]. The pain reduction in this case report illustrated the analgesic effects of acupuncture and I applaud the success in pain treatment and functional improvement observed at 6 weeks. Therefore, for patients diagnosed with CPSP, the use of acupuncture coupled with physical therapy and oral analgesics might be a useful combined treatment strategy. On the research front, I agree future high-quality randomized control trials with the three arms of acupuncture, i.e., acupuncture with conventional therapy, sham acupuncture with conventional therapy, and only conventional therapy, might further reveal the therapeutic effectiveness of acupuncture in reducing pain and restoring upper limb function.

Second, I support the authors' decision for not using the four acupoints in consideration of the patient's safety. Having said so, it is important for acupuncturists to realize that very few strict contraindications have been documented for the use of acupuncture for various therapeutic aims. Therefore, it is paramount that acupuncturists exercise appropriate clinical judgement in conducting the acupuncture treatment and the choice of the acupuncture points [4]. Despite the lack of strict contraindications, the authors did exercise appropriate clinical judgement and adopt a flexible approach in choosing the acupuncture points, seeking the contralateral side of the body as an alternative acupuncture treatment site. Contralateral acupuncture is also known as opposite needling or cross needling. At present, evidence pertaining to the use of contralateral acupuncture being superior to ipsilateral acupuncture in terms of the overall treatment outcome of post-stroke hemiplegic patients remains limited.

To reduce the risk of hypoesthesia of stroke with use of acupuncture in limbs, scalp acupuncture can be considered and has been reported in the use of CPSP [5]. Scalp acupuncture points described include the Ding zone and Dingqian zone. The application of electroacupuncture at the contralateral limb could also be considered for the treatment of neuropathic pain [5]. Electroacupuncture points used previously include LI15, SI9, SI11, and LE14 [5]. It might be possible to consider the use of scalp acupuncture points and limb electroacupuncture in future cases and assess if these strategies lead to more reduction in pain scores pre- and posttreatments.

I congratulate the authors for their success in treating this clinically challenging case and publication of this case report.

\section{CONFLICT OF INTEREST}

The author declares no conflict of interest.

\section{ORCID}

Yeow Leng Tan, https://orcid.org/0000-0003-0714-224X

\section{REFERENCES}

1. Gan JH, Santorelli LA. Acupuncture in post-stroke shoulder pain syndrome with multiple sclerosis: a case study. J Acupunct Meridian Stud 2021;14:27-31.

2. Osterberg A, Boivie J, Thuomas KA. Central pain in multiple sclerosis: prevalence and clinical characteristics. Eur J Pain 2005;9:531-42.

3. Lai HC, Lin YW, Hsieh CL. Acupuncture-analgesia-mediated alleviation of central sensitization. Evid Based Complement Alternat Med 2019;2019:6173412.

4. Van Hal M, Dydyk AM, Green MS. Acupuncture. [Updated 2021 Oct 24]. In: StatPearls [Internet]. Treasure Island (FL): StatPearls Publishing; 2021. Available from: https://www.ncbi. nlm.nih.gov/books/NBK532287/ 
5. Lee JA, Park SW, Hwang PW, Lim SM, Kook S, Choi KI, et al. Acupuncture for shoulder pain after stroke: a systematic review. J Altern Complement Med 2012;18:818-23.
Correspondence to. Yeow Leng Tan Department of Rehabilitation Medicine,

Singapore General Hospital, Singapore

E-mail: tanyeowleng2010@gmail.com

Received June 5, 2021, Accepted August 17, 2021

\section{Response to the Article "Comments on Acupuncture on Post-Stroke Shoulder Pain Syndrome with Multiple Sclerosis: A Case Study"}

\section{Dear Editor,}

We would like to thank Dr. Tan Yeow Leng from Singapore General Hospital for the encouraging comments on our case study titled "Acupuncture in Post-Stroke Shoulder Pain Syndrome with Multiple Sclerosis: A Case Study" [1]. It is heart-warming to hear a positive review from a colleague from such a prestigious hospital where one of us (Jia Hui Gan) had the opportunity to carry out her clinical placement as a physiotherapy student back in 2008.

We appreciate that Dr. Tan suggested the application of scalp acupuncture for this case study as it is commonly prescribed with positive results in cases with stroke. Alternatively, painless and imperceptible auricular acupuncture can also be offered to patients, which helps acknowledge that this craniofacial micro-acupuncture could be useful as a supplementary motor rehabilitation strategy instead of merely targeting at pain reduction post-stroke [2].

Regrettably, the studied subject did not agree to try scalp or auricular acupuncture. Since this was her first experience with acupuncture, she requested only conventional acupuncture. Subjects' rejection of treatments is not uncommon, especially if they have not been previously exposed to acupuncture. Patient's perception of acupuncture as an alternative medicine without scientific evidence still remains common, especially in the West, as reported by the first author (Jia Hui Gan) who has worked in the health sector both in Singapore and the United Kingdom. Therefore, prior to offering more specialised add-on acupuncture treatment to patients who are receiving acupuncture for the first time, it should be taken into account whether conventional acupuncture treatment can lead to improved pain outcomes in case of shoulder pain in patients with stroke [3].

We do agree with Dr. Tan's recommendation on applying electroacupuncture in central post-stroke pain (CPSP) as has been shown in a meta-analysis of acupuncture for post-stroke shoulder-hand syndrome [4]. This add-on acupuncture stimulation could also be applied on CPSP with complex past medical history, other than multiple sclerosis, such as heart disease, cancer, neuro-degenerative conditions, and autoimmune diseases. Early application of electroacupuncture could enhance Deqi gain by removing the blockages of vital energy in the meridians, especially in cases like the subject of this case study, whose Deqi were not attained easily [5]. We agree that we should have mentioned in our manuscript that the subject did not consent to electroacupuncture, scalp acupuncture, or auricular acupuncture, even when offered; instead, she agreed on the more common acupuncture treatment over torso or limbs only. This hesitation could be due to lack of integration of acupuncture treatment into day-to-day Western medicine practice, which still perceives acupuncture as an alternative medicine. It should also be acknowledged that acupuncture has still not been standardised as a mainstream pain treatment modality in the United Kingdom National Health System, which could explain the perception of add-on acupuncture treatments as "very invasive."

Regarding the proposed three-arm randomized controlled trials in CPSP by Dr. Tan, we would like to advocate that personal acceptance factors and subgroup analysis on different types of add-on acupunctures treatment should be taken into consideration to enhance the rigorousness of the study design.

Whilst waiting for more evidence to emerge to support acupuncture in managing CPSP, clinical judgement should be executed diligently to facilitate early recovery in complex CPSP cases that do not respond to conventional pain management. In addition, we believe that patients should be provided with in-depth health education about the beneficial effects of acupuncture treatment backed with scientific evidence to increase its acceptance in the treatment of CPSP. 


\section{CONFLICT OF INTEREST}

The author declares no conflict of interest.

\section{ORCID}

Jia Hui Gan,

https://orcid.org/0000-0003-4122-3166 Lorenzo A Santorelli,

https://orcid.org/0000-0002-8392-801X

\section{REFERENCES}

1. Gan JH, Santorelli LA. Acupuncture in post-stroke shoulder pain syndrome with multiple sclerosis: a case study. J Acupunct Meridian Stud 2021;14:27-31.

2. Miao D, Lei KT, Jiang JF, Wang XJ, Wang H, Liu XR, et al. Auricular intradermal acupuncture as a supplementary motor rehabilitation strategy in poststroke patients: a randomized preliminary clinical study. Evid Based Complement Alternat Med 2020;2020:5094914.
3. Chau JPC, Lo SHS, Yu X, Choi KC, Lau AYL, Wu JCY, et al. Effects of acupuncture on the recovery outcomes of stroke survivors with shoulder pain: a systematic review. Front Neurol 2018;9:30

4. Liu S, Zhang CS, Cai Y, Guo X, Zhang AL, Xue CC, et al. Acupuncture for post-stroke shoulder-hand syndrome: a systematic review and meta-analysis. Front Neurol 2019;10:433.

5. Lin C, Yuan HW, Zhang P, Liu YQ, Li CH, Li J, et al. [Review of studies on Deqi of acupuncture mainly in foreign countries]. Zhen Ci Yan Jiu 2013;38:168-73. Chinese.

Jia Hui Gan ${ }^{1, *}$, Lorenzo A Santorelli ${ }^{2}$ ${ }^{1}$ Inpatient Therapies Team, Haslemere Hospital, Royal Surrey NHS Foundation Trust, Surrey, United Kingdom, ${ }^{2}$ School of Biosciences and Medicine, University of Surrey, Surrey, United Kingdom

Correspondence to. Jia Hui Gan Inpatient Therapies Team, Haslemere Hospital, Royal Surrey NHS Foundation Trust, Surrey, United Kingdom

E-mail: jiahuigan1@gmail.com Received August 12, 2021, Accepted October 6, 2021 